\title{
Spatially antisymmetric localization of matter wave in a bichromatic optical lattice
}

\author{
Yongshan Cheng ${ }^{1,2 *}$ and S. K. Adhikari ${ }^{1 \dagger}$ \\ ${ }^{1}$ Instituto de Física Teórica, UNESP - Universidade Estadual Paulista, 01.140-070 São Paulo, São Paulo, Brazil \\ ${ }^{2}$ Department of Physics, Hubei Normal University, Huangshi 435002, People's Republic of China
}

\begin{abstract}
By direct numerical simulation of the time-dependent Gross-Pitaevskii equation using the splitstep Fourier spectral method we study the double-humped localization of a cigar-shaped BoseEinstein condensate (BEC) in a one-dimensional bichromatic quasi-periodic optical-lattice potential, as used in a recent experiment on the localization of a BEC [Roati et al., Nature 453, 895 (2008)]. Such states are spatially antisymmetric and are excited modes of Anderson localization. Where possible, we have compared the numerical results with a variational analysis. We also demonstrate the stability of the localized double-humped BEC states under small perturbation.
\end{abstract}

PACS numbers: 03.75.Nt,03.75.Lm,64.60.Cn,67.85.Hj

\section{INTRODUCTION}

Fifty years after the prediction of Anderson localization [1] of electron wave in a disorder potential, the recent experimental localization [2, 3] of a non-interacting cigar-shaped Bose-Einstein condensate (BEC) in a quasiperiodic bichromatic optical-lattice (OL) [4 22] and speckle [23] potentials has drawn much attention of research workers. The quasi-periodic bichromatic OL potential [5] used in the localization of a non-interacting BEC [4] was formed by the superposition of two standingwave polarized laser beams with incommensurate wavelengths.

Recently, there has been studies of localized BECs with a maximum at the center of the bichromatic OL trap, where the potential is a minimum [10, 12 14]. A natural extension of this phenomenon would be to investigate localization in more exotic situations, e.g., where a minimum of the localized BEC is created at the center of the trap. Here, with numerical simulation of the GrossPitaevskii (GP) equation, we study the double-humped spatially-antisymmetric localization in an excited state [24 29] of a cigar-shaped BEC in a one-dimensional (1D) bichromatic quasi-periodic OL potential. The two humps appear in a single site of the OL potential. Doublehumped structures have been created in a BEC by phase imprinting and other methods 30, 31] and also have been studied theoretically 32 35]. This makes the present investigation also of experimental interest. Anderson localization was originally predicted for the non-interacting system. However, in the present study on the localization of a double-humped BEC we also consider a weaklyinteracting system. Although, the present localization is very similar to Anderson localization in a fully disordered potential, the bichromatic OL potential is quasi periodic and hence deterministic in nature. The localization considered here is well described by the 1D discrete AubryAndré model of quasi-periodic confinement [36, 37].

\footnotetext{
*yong_shan@163.com

†adhikari@ift.unesp.br; URL: www.ift.unesp.br/users/adhikari
}
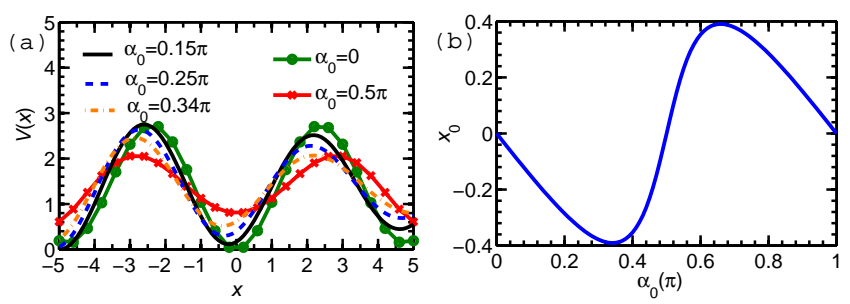

FIG. 1: (Color online) (a) The bichromatic OL potential $V(x)$ vs. $x$ for different phases $\alpha_{0}$. (b) The position $x_{0}$ of the OL's central minimum vs. $\alpha_{0}$. Potential parameters in Figs. 1 to 7 are $\lambda_{1}=10, \lambda_{2}=0.862, \lambda_{1}, s_{1}=10$ and $s_{2}=3$.

If the bichromatic OL potential $V(x)$ has the symmetry $V(x)=V(-x)$, the localized states $\phi(x)$ of the non-interacting BEC has the symmetry $\phi(x)= \pm \phi(-x)$. Here we consider spatially antisymmetric localized states satisfying $\phi(x)=-\phi(-x)$, which should be considered to be excited modes [24] of Anderson localization.

In the presence of strong disorder, the localized state could be quite similar to a localized state of Gaussian shape in an infinite potential. However, the more interesting case of localization is in the presence of a weak disorder when the system is localized due to the quasiperiodic nature of the potential [4, 23] and not due to the strength of the lattice. When this happens the chemical potential of the system becomes comparable to the height of the bichromatic lattice and the localized state develops an exponential tail. Nevertheless, the central part of the localized BEC is found to have a modulated Gaussian shape which allows the consideration of a variational approximation.

\section{ANALYTICAL CONSIDERATION}

We consider a cigar-shaped BEC under tight transverse confinement with the bichromatic OL acting along the axial $x$ direction. Then it is appropriate to consider a $1 \mathrm{D}$ reduction of the three-dimensional GP equation by 


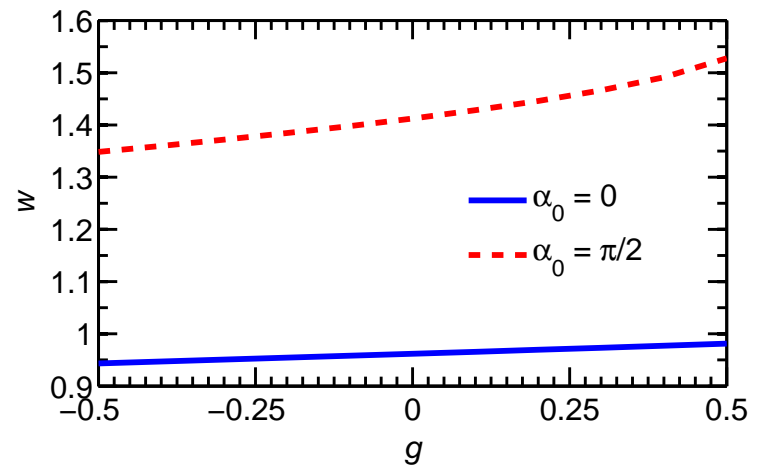

FIG. 2: (Color online) The pulse interval $w$ of the localized state vs. the non-linearity $g$ for different phase $\alpha_{0}$.

freezing the transverse dynamics to the respective ground state and integrating over the transverse variables. The double-humped localized state of $N$ atoms can be described by the dimensionless GP equation [38, 39]

$$
i \frac{\partial u}{\partial t}=-\frac{1}{2} \frac{\partial^{2} u}{\partial x^{2}}+g|u|^{2} u+V(x) u,
$$

with normalization $\int_{-\infty}^{\infty}|u|^{2} d x=1$, of the BEC wave function $u \equiv u(x, t)$. The spatial variable $x$, time $t$, and energy are expressed in transverse harmonic oscillator units $a_{\perp}=\sqrt{\hbar /(m \omega)}, \omega^{-1}$ and $\hbar \omega$, where $m$ is the mass of an atom and $\omega$ is the angular frequency of the transverse trap with non-linearity [38] $g=2 a N / a_{\perp}^{2}$, and $a$ the atomic scattering length. The bichromatic OL potential $V(x)$ is taken as:

$$
V(x)=\sum_{l=1}^{2} A_{l} \sin ^{2}\left(k_{l} x+\alpha_{l}\right)
$$

with $A_{l}=2 \pi^{2} s_{l} / \lambda_{l}^{2},(l=1,2)$, where $\lambda_{l}$ 's are the wavelengths of the OL potentials, $s_{l}$ are their intensities, $\alpha_{l}$ are phases, and $k_{l}=2 \pi / \lambda_{l}$ the wave numbers. We take the phase of the first OL $\alpha_{1}=0$ and that of the second $\alpha_{2} \equiv \alpha_{0}$. In this investigation, we take the ratios $\lambda_{2} / \lambda_{1}=0.862$ and $s_{2} / s_{1}=0.3$ which are roughly the same as in the experiment [4]. We further take $\lambda_{1}=10$, and $s_{1}=10$. The experiment of [4] employed similar strengths of the optical lattice to study Anderson localization in weak disorder.

For phase difference $\alpha_{0}=0$, potential (2) has a minimum at $x=0$. The position of this minimum moves to $x=x_{0} \neq 0$ for $\alpha_{0} \neq 0$. We show in Fig. 1 (a) the bichromatic OL potential (2) as $\alpha_{0}$ is varied. The bichromatic OL potential is symmetric around $x=0$ when $\alpha_{0}=0$ or $\pi / 2$. For the phase $\alpha_{0}$ in the range $0<\alpha_{0}<\pi / 2$, the OL potential is asymmetric, as shown in Fig. 1 (a). In Fig. 1 (b) we show the position of the central minimum of potential (2) as $\alpha_{0}$ is changed.

For a variational analysis of the localized state we consider the stationary wave form $\phi(x)$ given by $u(x, t)=$ $\exp (-i \mu t) \phi(x)$, where $\mu$ is the chemical potential. The real wave function, $\phi(x)$, obeys the stationary equation,

$$
\mu \phi(x)+\phi^{\prime \prime}(x) / 2-g \phi^{3}(x)-V(x) \phi(x)=0,
$$

where the prime denotes space derivative. For $\alpha_{0}=0$ or $\pi / 2$, potential (2) leads to spatially-symmetric or antisymmetric states confined practically to the central site of the quasi-periodic OL potential. When this happens, a variational approximation with the following Gauss-type ansatz is useful for the spatially-antisymmetric state

$$
\phi(x)=\frac{1}{\pi^{1 / 4}} \sqrt{\frac{2 \mathcal{N}}{w^{3}}} x \exp \left[-\frac{x^{2}}{2 w^{2}}\right],
$$

where $w$ is the spatial extension of the localized state and will be termed pulse interval of the localized BEC centered at $x=0$, and $\mathcal{N}$ is the normalization. The Lagrangian of the system is given by

$$
\begin{aligned}
L & =\int_{-\infty}^{\infty}\left[\mu \phi^{2}-\left(\phi^{\prime}\right)^{2} / 2-g \phi^{4} / 2-V(x) \phi^{2}\right] d x-\mu, \\
& =\mu \mathcal{N}-\frac{3 \mathcal{N}}{4 w^{2}}-\frac{3 g \mathcal{N}^{2}}{8 \sqrt{2 \pi} w}-\mu-\mathcal{N} \sum_{l=1}^{2} A_{l} L_{l} \\
L_{l} & =\left[\frac{1}{2}+\left(k_{l}^{2} w^{2}-\frac{1}{2}\right) \cos \left(2 \alpha_{l}\right) \exp \left(-k_{l}^{2} w^{2}\right)\right] .
\end{aligned}
$$

The first variational equation $\partial L / \partial \mu=0$ fixes the normalization: $\mathcal{N}=1$. We use it in the following equations. The remaining equations $\partial L / \partial w=\partial L / \partial \mathcal{N}=0$ yield, respectively,

$$
\begin{aligned}
1 & =\frac{4 w^{4}}{3} \sum_{l=1}^{2} A_{l} k_{l}^{2}\left(\frac{3}{2}-k_{l}^{2} w^{2}\right) \cos \left(2 \alpha_{l}\right) \exp \left(-k_{l}^{2} w^{2}\right) \\
& -\frac{g w}{4 \sqrt{2 \pi}} \\
\mu & =\frac{3}{4 w^{2}}+\frac{3 g}{4 w \sqrt{2 \pi}}+\sum_{l=1}^{2} A_{l} L_{l} .
\end{aligned}
$$

Equation (7) determines the pulse interval of the localized state. The corresponding energies are given by $E=\int\left[\left(\phi^{\prime}\right)^{2} / 2+g \phi^{4} / 2+V \phi^{2}\right] d x=\mu-3 g /(8 w \sqrt{2 \pi})$.

In Fig. 22 we plot the variational results for the pulse interval $w$ vs. the nonlinear coefficient $g$ according to Eq. (17). The pulse interval becomes larger as $g$ changes from negative (attractive) to positive (repulsive). The reason is that the repulsive interaction among atoms induces a macroscopic repulsion between the two constituent pulses thus increasing the pulse interval. Compared with $\alpha_{0}=$ 0, in Fig. 2. for $\alpha_{0}=\pi / 2$ the pulse interval is larger and increases faster with $g$. This is because, for $\alpha_{0}=\pi / 2$, the trapping is weaker relative to $\alpha_{0}=0$.

\section{NUMERICAL RESULTS}

We perform the numerical simulation employing the real-time split-step Fourier spectral method with space 

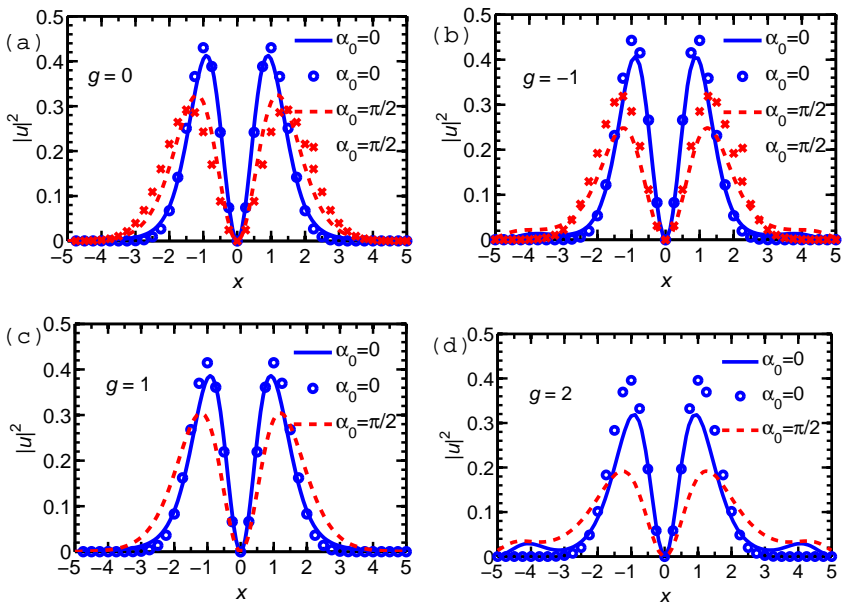

FIG. 3: (Color online) Numerical (line) and variational (symbol) density $|u|^{2}$ of the antisymmetric double-humped BEC vs. $x$ for (a) $g=0$, (b) $g=-1$, (c) $g=1$, and (d) $g=2$.

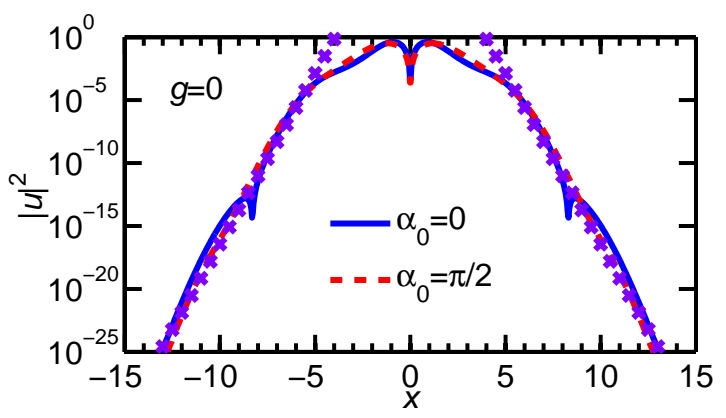

FIG. 4: (Color online) Numerical density $|u|^{2}$ of localized states of Fig. 3 (a) vs. $x$ in log scale. The crosses are exponential fit $\sim \exp (-|x| / d)$ to density, with localization length $d=0.17$.

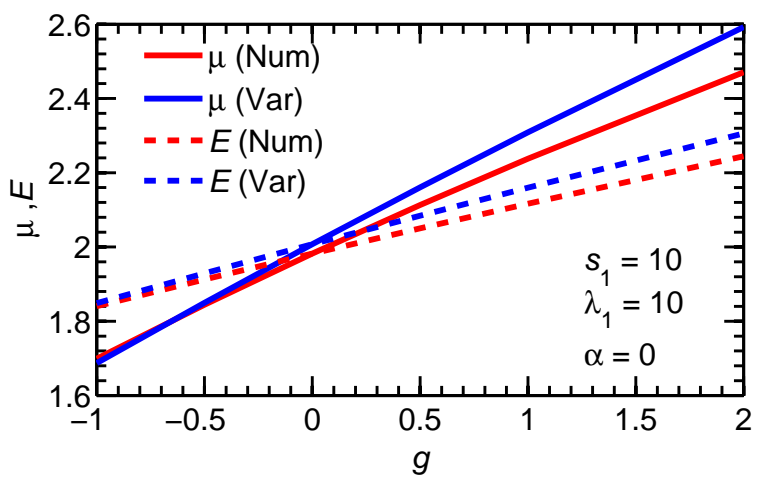

FIG. 5: (Color online) Numerical (Num) and variational (Var) energies and chemical potential vs. nonlinearity $g$.
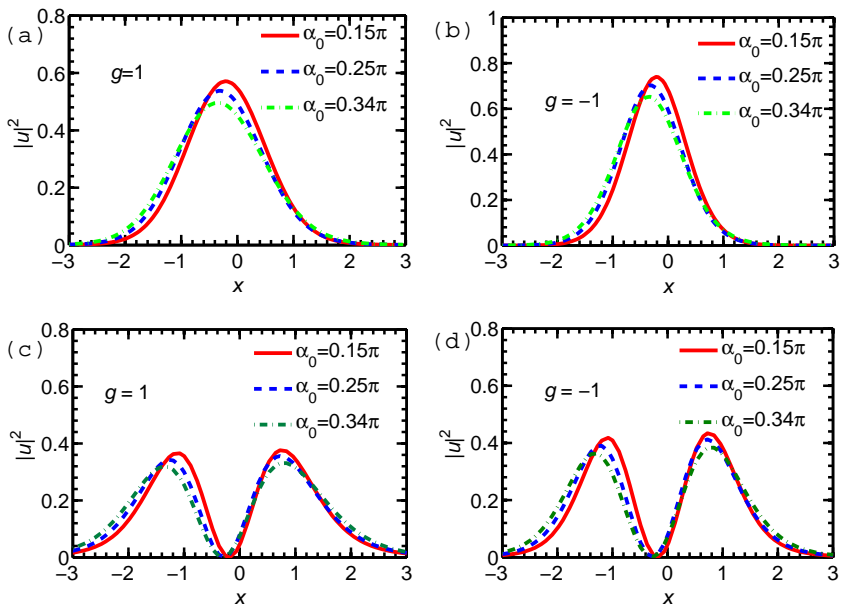

FIG. 6: (Color online) Numerical density of the asymmetric single-humped BEC vs. $\mathrm{x}$ for different $\alpha_{0}$ for (a) $g=1$ and (b) $g=-1$. (c) and (d) represent double-humped results for $g=1$ and -1 , respectively.

step 0.04, time step 0.0001. Although we use a timedependent approach the localized states we calculate are stationary in nature. The initial input pulse is taken as $u(x, 0)=\sqrt{2} x \exp \left(-x^{2} / 2\right) / \pi^{1 / 4}$ with a parabolic trap $V^{\prime}(x)=x^{2} / 2$ and $g=0$. In the beginning, during time evolution the parabolic trap is slowly turned off and the bichromatic OL is slowly turned on by increasing $s_{1}$ by 0.00001 in each time step. Successively, during time evolution we change gradually the nonlinear coefficient $g$ by 0.00001 in each time step to obtain the stable localized states.

Figures 3 (a), (b), (c), and (d) illustrate typical numerical and variational profiles of the localized states for $g=0,-1,1$ and 2 for $\alpha_{0}=0$ and $\pi / 2$. From Figs. 3 we find that the numerical densities are in good agreement with the variational results for small non-linearity $g$. If $g$ is larger or the trapping is weaker, however, the localized states develop undulating tails and occupy more than one OL site. In that case, density envelope of the localized state can not be described well by Eq. (4) and the variational approximation will no longer be as good. We also calculated the chemical potential and energy of these states using Eqs. (77) and (8). The results are shown in Fig. 5. From Fig. 5 we find that the energy values are comparable to the trapping potential shown in Fig. 11 (a). This guarantees the interesting limit of weak disorder as emphasized in [4, 23].

To see this weak disorder explicitly, we plot in Fig. 4 the density $|u|^{2}$, in log scale, of the localized states shown in Fig. 3 (a). The long exponential tail extends from $x=13$ to -13 , whereas the central part of density distribution in Fig. 3 (a) contributing to normalization is limited between $x \approx \pm 5$. We have also shown in Fig. 4 the exponential fit to density $\sim \exp (-|x| / d)$ with the localization length [23] $d=0.17$. 
(a)

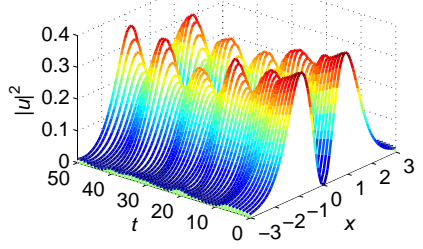

(b)

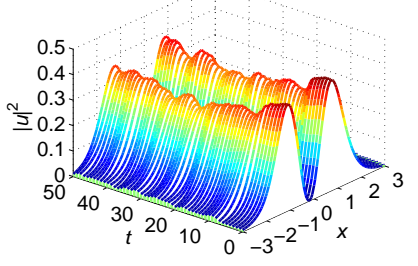

FIG. 7: (Color online) Stability dynamics $|u(x, t)|^{2}$ vs. $x$ and $t$ for (a) the antisymmetric state with $g=1, \alpha_{0}=0$, [viz. Fig. 3 (c)] as the center of the OL trap is displaced by a small distance $\Delta x=0.1$ at time $t=5$, and for (b) the asymmetric state with $g=-1, \alpha_{0}=0.15 \pi$ [viz. Fig. 6] (d)] as the wave length $\lambda_{1}$ is suddenly changed from 10 to 11 at time $t=5$.

Next we present numerical analysis for the asymmetrical OL potential (2) with $\alpha_{0} \neq 0$ or $\neq \pi / 2$. As shown in Fig. 11 (b), the position of the OL's minimum is no more at $x=0$. On the other hand, the displacement of the OL's minimum changes periodically as $\alpha_{0}$ increases. Because of the periodicity, we restrict our attention to $0<\alpha_{0}<\pi / 2$. In this case, to avoid the loss of accuracy in the localized state in the numerical integration, we move the center of the initial input pulse and the parabolic trap to the position of the OL's minimum. Thus we calculate the profiles of the localized states in the asymmetrical OL potential for $g=1,-1$, and $\alpha_{0}=0.15 \pi, 0.25 \pi$, and $0.34 \pi$ and show the results in Fig. 6. In order to understand the novel effect, we first investigate the single-humped localized BECs with the asymmetrical OL potential calculated using the input pulse $u(x, 0)=\exp \left(-x^{2} / 2\right) / \pi^{1 / 4}$. The results are shown in Figs. 6 (a) and (b) for $g=1$ and -1 , respectively. It can be seen that the atom density envelopes of the single-hump localized BEC are asymmetrical. Compared with Fig. 6 (a), Fig. 6] (b) presents narrower and larger atom densities because of the focusing nature of the BEC interaction $(g=-1)$. When $\alpha_{0}=0.34 \pi$, the pulse width of the localized state is larger compared to the widths for $\alpha_{0}=0.15 \pi$, or $0.25 \pi$. The reason is that the overall trapping is weaker as $\alpha_{0}$ increases.

The same results are next obtained for the doublehumped localized BECs which are shown in Figs. 6 (c) and (d) for $g=1$ and $g=-1$, respectively, using the initial input pulse $u(x, 0)=\sqrt{2} x \exp \left(-x^{2} / 2\right) / \pi^{1 / 4}$. These two Figs. show the asymmetry of the two humps for the double-humped localized BECs. The right hump of the localized states is higher than its left hump because trapping on the left is stronger. When $\alpha_{0}=0.34 \pi$, the difference in the height of the two humps and the pulse interval of the localized state are larger compared with those of $\alpha_{0}=0.25 \pi$ or $0.15 \pi$. These results are in agreement with single-humped localized BECs illustrated in Figs. 6 (a) and (b).

One of the most important issues is the stability of the double-humped localization against perturbation. First,

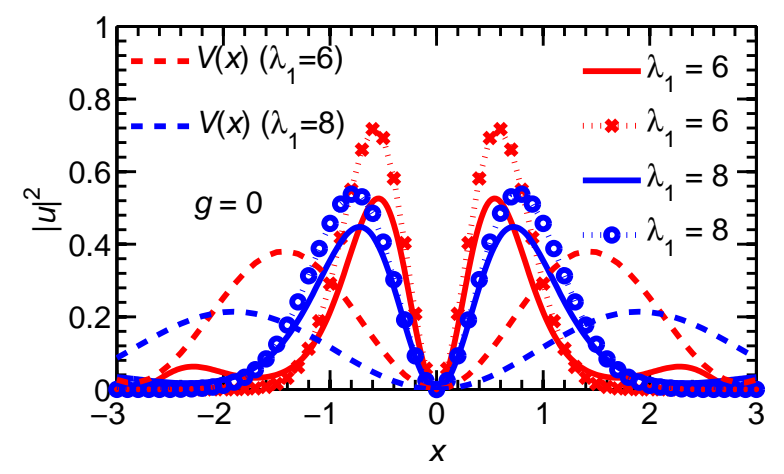

FIG. 8: (Color online) The numerical (solid line) and variational (dotted line with symbol) density $|u|^{2}$ vs. $x$ of the double-humped BEC for $\lambda_{1}=6$ and 8 , and $g=0, s_{1}=$ $10, s_{2}=3, \lambda_{2}=0.862 \lambda_{1}$, and $\alpha_{0}=0$. The corresponding bichromatic OL potential is also shown in arbitrary units.

we investigate the stability of the antisymmetrical localization. In order to examine whether the predicted localized state is stable, we introduce a small perturbation from equilibrium point by displacing the center of the OL trap by a small distance $\Delta x=0.1$ at $t=5$. The numerical simulations are shown in Fig. 7 (a) for $g=1, \alpha_{0}=0$. The double-humped localization oscillate around the new center of the OL trap, and the symmetry of the two humps is broken after the small perturbation is introduced. The double-humped localization exists during a large time interval for small perturbation. We also checked the stability of the states for other values (negative) of $g$ and for other types of perturbation by changing the value of the wave length $\lambda_{1}$ and the antisymmetrical localized state was found to be stable. Next we studied the stability of the asymmetric localized states. The stability of one such state is shown in Fig. 7 (b) for $\alpha_{0}=0.15 \pi$ and $g=-1$ when $\lambda_{1}$ was suddenly changed from 10 to 11 at time $t=5$. The double-humped state is again found to be stable against small perturbation.

So far we considered a fixed OL wave length: $\lambda_{1}=10$. To get insight into the effects of the OL on the stationary localized states, we now investigate the properties of the system when the wave lengths $\lambda_{l}$ are smaller. In realistic experiment, the dimensionless wavelength can be adjusted by the harmonic trap because $\lambda_{1}$ is related to the transverse harmonic-oscillator length. The wave length $\lambda_{1}$ controls the dimensionless heights of potential (2), so it is meaningful to investigate the effects of $\lambda_{1}$ on the stationary localized states. In this part, the same parameters of the numerical integration are selected as those in the preceding calculation, (viz. $s_{1}=10, s_{2}=3$, $\lambda_{2}=0.862 \lambda_{1}$, and $\left.\alpha_{0}=0\right)$. In Fig. 8 we plot $|u|^{2}$ vs. $x$ for $g=0$ and different $\lambda_{1}$. A smaller $\lambda_{1}$ leads to a localized state with a smaller pulse interval $w$. We have also compared the numerical results with variational analysis for small $\lambda_{1}$. The variational results are shown by the 
chain of symbols in Fig. 8

\section{SUMMARY}

Using the numerical and variational solution of the GP equation, we studied the stationary localization of a double-humped cigar-shaped BEC in a bichromatic quasi-periodic 1D OL potential. The bichromatic OL potential is generated by superposing two OL potentials in the form of sine waves. Such a bichromatic OL potential is symmetric around the center at $x=0$, consequently, the density of the localized state also possesses the same symmetry. In the presence of a phase difference between the two OL components the above symmetry is broken and we analyze this symmetry breaking in case of singlehumped and double-humped states. Here we also study the effect of a weak atomic interaction (both attractive and repulsive) on the profile of the localized states. The localized double-humped states were found to be dynamically stable under small perturbations. We hope that the present work will motivate new studies, specially experimental ones on Anderson localization in the form of double-humped states.

\section{Acknowledgments}

FAPESP and CNPq (Brazil) provided partial support.
[1] P. W. Anderson, Phys. Rev. 109, 1492 (1958).

[2] J. Chabé, G. Lemarié, B. Grémaud, D. Delande, P. Szriftgiser, and J. C. Garreau, Phys. Rev. Lett. 101, 255702 (2008).

[3] E. E. Edwards, M. Beeler, T. Hong, and S. L. Rolston, Phys. Rev. Lett. 101, 260402 (2008).

[4] G. Roati et al., Nature 453, 895 (2008).

[5] V. I. Yukalov, Laser Phys. 19, 1 (2009).

[6] L. Sanchez-Palencia, D. Clément, P. Lugan, P. Bouyer, G. V. Shlyapnikov, and A. Aspect, Phys. Rev. Lett. 98, 210401 (2007).

[7] J. E. Lye, L. Fallani, M. Modugno, D. S. Wiersma, C. Fort, and M. Inguscio, Phys. Rev. Lett. 95, 070401 (2005).

[8] B. Damski, J. Zakrzewski, L. Santos, P. Zoller, and M. Lewenstein, Phys. Rev. Lett. 91, 080403 (2003).

[9] T. Schulte, S. Drenkelforth, J. Kruse, W. Ertmer, J. Arlt, K. Sacha, J. Zakrzewski, and M. Lewenstein, Phys. Rev. Lett. 95, 170411 (2005).

[10] M. Modugno, New. J. Phys. 11, 033023 (2009).

[11] M. Larcher, F. Dalfovo, and M. Modugno, Phys. Rev. A 80, 053606 (2009).

[12] S. K. Adhikari and L. Salasnich, Phys. Rev. A 80, 023606 (2009).

[13] Y. Cheng and S. K. Adhikari, Phys. Rev. A 81, 023620 (2010).

[14] S. K. Adhikari, Phys. Rev. A 81, 043636 (2010).

[15] A. S. Pikovsky and D. L. Shepelyansky, Phys. Rev. Lett. 100, 094101 (2008).

[16] S. Flach, D. O. Krimer, and Ch. Skokos, Phys. Rev. Lett. 102, 024101 (2009).

[17] T. Paul, P. Schlagheck, P. Leboeuf, and N. Pavloff, Phys. Rev. Lett. 98, 210602 (2007).

[18] I. García-Mata and D. L. Shepelyansky, Phys. Rev. E 79, 026205 (2009).

[19] Ch. Skokos, D. O. Krimer, S. Komineas, and S. Flach,
Phys. Rev. E 79, 056211 (2009).

[20] T. Roscilde, Phys. Rev. A 77, 063605 (2008).

[21] T. Paul, M. Albert, P. Schlagheck, P. Leboeuf, and N. Pavloff, Phys. Rev. A 80, 033615 (2009).

[22] A. Zenesini, H. Lignier, C. Sias, O. Morsch, D. Ciampini and E. Arimondo, Laser Physics 20, 1182 (2010).

[23] J. Billy et al., Nature 453, 891 (2008).

[24] V. I. Yukalov and V. S. Bagnato, Laser Phys. Lett. 6, 235 (2009).

[25] V. I. Yukalov and V. S. Bagnato, Laser Phys. Lett. 6, 399 (2009).

[26] V. I. Yukalov, Laser Phys. Lett. 7, 467 (2010).

[27] V. I. Yukalov, Laser Phys. Lett. 6, 833 (2009).

[28] V. I. Yukalov, Laser Phys. Lett. 6, 688 (2009).

[29] S. K. Adhikari, Laser Phys. Lett. 6, 901 (2009).

[30] S. Burger et al., Phys. Rev. Lett. 83, 5198 (1999).

[31] B. P. Anderson, P. C. Haljan, C. A. Regal, D. L. Feder, L. A. Collins, C. W. Clark, and E. A. Cornell, Phys. Rev. Lett. 86, 2926 (2001).

[32] T. Busch and J. R. Anglin, Phys. Rev. Lett. 84, 2298 (2000).

[33] G. V. Shlyapnikov, W. Ertmer, K. Sengstock, and M. Lewenstein, Phys. Rev. Lett. 89, 110401 (2002).

[34] S. K. Adhikari, J. Low Temp. Phys. 143, 267 (2006).

[35] P. G. Kevrekidis, R. Carretero-Gonzalez, G. Theocharis, D. J. Frantzeskakis, and B. A. Malomed, Phys. Rev. A 68, 035602 (2003).

[36] S. Aubry and G. André, Ann. Israel Phys. Soc. 3, 33 (1980).

[37] J. Biddle, B. Wang, D. J. Priour, Jr., and S. Das Sarma, Phys. Rev. A 80, 021603(R) (2009).

[38] C. A. G. Buitrago and S. K. Adhikari, J. Phys. B 42, 215306 (2009).

[39] L. Salasnich, A. Parola, and L. Reatto, Phys. Rev. A 65, 043614 (2002). 


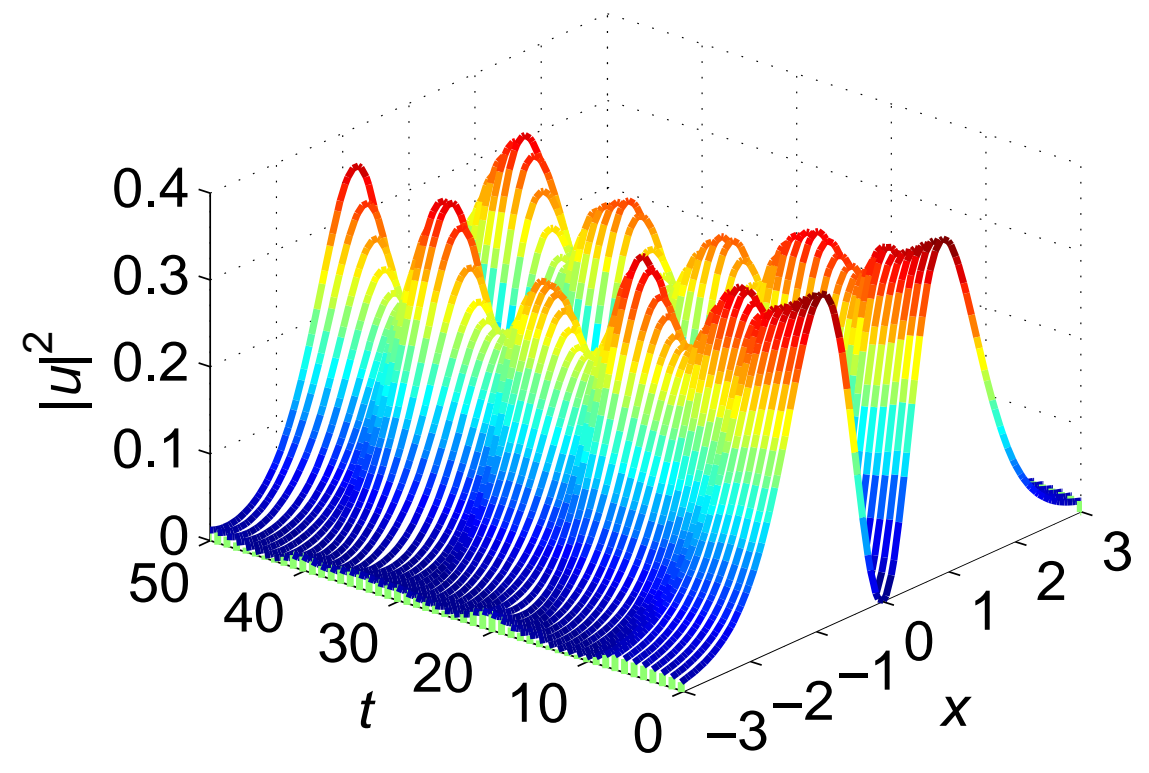


Laser Physics

Letters 